scleral incision. With this method only monocular vision is possible with all its limitations, such as attempting to judge orientation, and considerable stirring up of the vitreous. must occur in most cases. For this reason we believe the first method is the operation of choice.

For the extraction, the forceps illustrated is used (Fig. 24 a open; Fig. 24 b closed).

\title{
Summary
}

A new localiser and method of localisation of radio-opaque intraocular F.B.'s is described.

An improved operative technique is described in stages.

The problems of diathermy and magnetism are discussed and advice offered on how to regulate and use the diathermy apparatus to avoid complications.

A diagram of new forceps for extraction of non-magnetic F.B.'s is given.

We wish to thank Colonel A. J. Gibson, D.S.O., T.D., Officer Commanding the Hospital for granting us facilities and help in our work, and Lieut.-Colonel G. I. Scott, Adviser in Ophthalmology, M.E.F., for permission to publish the paper.

We are greatly indebted to Professor Goldberg and his Staff of the Experimental Laboratory, Tel-Aviv, for advice in solving technical problems.

Lastly, to the Staff and Workmen of 309 L.O.C. Workshops, R.E.M.E., our sincere thanks are due for the construction of the localiser and their practical advice in interpreting the design.

\section{WAR SURGERY OF THE EYE IN FORWARD AREAS*}

BY

\author{
Major E. C. ZORAB, R.A.M.C. \\ SURGEON TO A MOBILE OPHTHALMIC UNIT
}

IN ophthalmic surgery, as in general surgery great advances have been made in the facilities which are available to a man wounded in battle.

Every eye injury comes under the care of what one may call an ophthalmic team, which consists of three parts, viz.,

(1) A Mobile Ophthalmic Unit.

(2) A Base Ophthalmic Wing.

(3) An Ophthalmic Department in United Kingdom, perhaps St. Dunstan's.

* Received for publication, April 30, 1945. 
In order to appreciate the type of work done by each part of the team, one should have a clear picture of the conditions under which work is done, and the lines of communication from battlefield to base. These conditions vary with the seasons and with the types of terrain, and have a definite bearing on the method of treating a case.

Let us follow the case of a man wounded in the eye during an attack. If it is an eye injury only, he will make his own way back to the R.A.P. If his eye wound is one of many wounds which prevent him walking he will wait until collected by stretcher bearers who will carry him back there. Here he will be seen by the R.M.O., dressed, given a hypodermic injection of morphia gr. $1 / 4$, and started on a course of oral sulphanilamide. His next journey is by ambulance (which may be the ordinary type or Jeep, or even mule) to the A.D.S. of the Field Ambulance. Here resuscitation measures are applied if necessary and he is sent on down to the M.D.S.

This is the "Sorting Centre" and from here he will be sent either to the Advanced Surgical Centre located at an F.D.S. for priority one treatment of his general wounds, or direct to the forward C.C.S. in the Corps at which will be located the Mobile Ophthalmic Unit for that Corps. He may have other wounds requiring more immediate surgery and this will necessitate remaining at the F.D.S., but the majority of cases are fit to be evacuated to the Mobile Ophthalmic Unit before any general surgery is undertaken. Some cases, such as compound fractures of the femur, bleeding wounds, and other cases unfit to travel are operated on at the F.D.S. and sent on to the Ophthalmic Unit the following day. A few cases, such as abdominal perforations and chest wounds with haemorrhage need to be operated on at F.D.S. and remain there from ten to fourteen days. This necessitates the ophthalmic surgeon visiting the patient to assess the severity of the eye wound, possibly taking with him minimum operating equipment. The majority of cases therefore reach the Ophthalmic Unit between six or twelve hours after wounding, some twentyfour to thirty-six hours, and a few are visited perhaps twenty-four hours after wounding, but cannot be sent to any part of the ophthalmic team for ten to fourteen days or more.

The second stage of the journey through the hands of the ophthalmic team is nearly always a long one in distance but since air evacuation is usually available it is a comparatively short one in time. The fitness or otherwise for evacuation from the first to the second parts of the team therefore depends almost entirely on the accessibility of the airstrip to the forward C.C.S. Sometimes it is a matter of two or three miles over tar-mac roads, and at others fifteen to twenty miles over third-rate roads with many demolitions 
and diversions. In the former case a patient can often be evacuated without harm, forty-eight hours after operation, while in the latter it may be necessary to retain him for five or six days or even more.

- The third stage of the journey depends on the available space in hospital ships returning to the United Kingdom; except in the case of blind patients for whom priority can be obtained either by sea or air, for transmission direct to St. Dunstan's.

Such then is the composition of the ophthalmic team; widely separate in distance, but working closely together for the same end. Here let it be said that it is a great advantage for the forward surgeon to know who is behind him, both personally and professionally. It makes for closer team work and helps in preventing him attempting too much or too little. It also allows him to know that his mistakes will be pointed out with fair criticism, and without malice, and that helpful suggestions will be made.

There is one great disadvantage to forward surgery, and that is that the surgeon never sees the end results of the cases on which he has operated. Follow-up cards help to some extent, but are never the same thing as seeing for oneself. In addition, it is often impossible to assess the end result for a year or even more, when all the usual dangers and complications may be said to have been passed, and perhaps several further operations performed.

For these reasons one cannot judge the adequacy or correctness of one's surgery by the visual result obtained either immediately or after a few weeks. Some months later the picture may have been changed vastly-for better or for worse. Therefore any attempt to produce statistics of operative results until all the cases have been carefully followed for more than a year, is bound to be fraught with difficulty and full of fallacies. For guidance, one must rely on one's own experience of early operative results, the long term experience of other surgeons, and the comments of the surgeons at the base.

\section{Method of working of the ophthalmic unit}

The surgeon is notified by the reception clerk as an " eye case" arrives. The same is left in reception until the surgeon, accompanied by his orderly, comes to see it. Unless he happens to be operating this will be immediately, day or night. The orderly brings with him the " examination tray" containing cotton wool swabs, wet and dry; pads; flaps; bandages; adhesive plaster; cocaine; atropine; sodium penicillin, drops or ointment; glass rods; Desmarres' retractor; ophthalmoscope and binocular loupe.

The case is diagnosed, and the Field Medical Card written up and re-attached_to the patient. It may be necessary to consult 
with the general surgeon and the resuscitation officer, with regard to other wounds or the patient's general condition. X-rays are taken if required and the man is then transferred to the preoperation ward to await operation, premedication having been ordered for a certain time. Unless contraindicated the patient is undressed, washed, put into pyjamas, and back on to his stretcher. When called for he is taken to the theatre, and put on the operating table, still on his stretcher.

This unit carries the full equipment of an ophthalmic operating theatre, including anaesthetic apparatus. It is preferable to have one's own theatre, but essential to have at least one's own table in a combined theatre. It is unsatisfactory to both parties for the ophthalmic surgeon to use the C.C.S. theatre for his cases, particularly if there are many general cases waiting as there usually are. It also means a lot of unnecessary carrying of instruments, magnet equipment, electric wiring, and much waiting for a vacant theatre.

The most convenient theatre, for the summer months at any rate, is the E.P.I.P. tent which is part of the equipment. As far as possible it has always been arranged for the ophthalmic operating theatre to be located within an easy stretcher carry from the general surgical theatre. If there have been only minor wounds in the rest of the patient they have been dealt with in the ophthalmic theatre, for which purpose a few extra instruments are carried. For more serious wounds the patient has been transferred, still under an anaesthetic, to the general theatre, or has had his other wounds treated first by the general surgeon and then been transferred for his eye operation.

For more than six months, I was associated at_various C.C.S.'s with the advanced sections of a maxillo-facial, and a neurosurgical unit, often sharing an operating theatre and an anaesthetist. This association, which came to be widely known as "The Trinity" was most satisfactory from all points of view. - It enabled all wounds occurring above the neck to be dealt with at one centre, with one anaesthetic, and, not the least important, it gave all three specialists valuable experience in other closely allied specialities. For what I learnt from this association, I shall always be grateful to Major H. V. Slemon, R.C.A.M.C., Major Murley, R.A.M.C., Major E. J. Dalling, A.D.C., and Major R. P. Harbord, R.A.M.C., the anaesthetist.

The type of anaesthesia was determined by the requirements of the particular case. This unit does not carry an anaesthetist for the very good reason that he would seldom be fully employed. In normal periods, the services of an anaesthetist can usually be borrowed for the small number of operations performed. During a battle, when an anaesthetist could be fully employed, the C.C.S. 
anaesthetists are too busy. Occasionally, one was attached for temporary duty from a non-working unit, buit often I had to give my own general anaesthetic-an unsatisfactory practice.

While working with the " Trinity" an anaesthetist was shared by all three units, and he frequently had three anaesthetics under supervision simultaneously. This arrangement was satisfactory only so long as the three teams were either sharing a large theatre, or were working in adjoining theatres. Nearly all jaw, face, and head cases that needed general anaesthesia needed endotracheal intubation, and could thereafter be safely left on the Oxford rapouriser under observation. Comparatively few eye casesless than 20 per cent.-could be done under local anaesthesia partly on account of other injuries requiring general anaesthesia, and partly because having only recently been wounded in battle, most patients were not in a fit condition psychologically to undergo an operation under local anaesthesia.

It was found that the anaesthetic of choice was a continuous pentothal drip. It was given as a $\frac{1}{2}$ per cent. solution in normal saline or glucose saline with an ordinary intravenous infusion set, combined with cocaine drops in the eye, morphia and hyoscine premedication; and continuous oxygen via an intranasal pharyngeal tube. This gave a very smooth anaesthetic with the minimum of trouble, practically no respiratory movements of the head, little or no post-anaesthetic cough or vomiting, and could be continued for as long as was required. As much as 3.5 grams of pentothal was given on occasions for long combined cases with no ill effect.

\section{Types of cases dealt with}

Injured eyes can be roughly divided into two classes-Nonrepairable and Repairable.

(I) Non-repairable.-Under this heading are included all eyes that are so badly damaged as to be anatomically non-repairable. In every case of course there is no perception of light. Their removal is not urgent and the indications for operation at this level are :-

(a) When other wounds necessitate a general anaesthetic. It is as well to remove the eye at the same time to avoid the necessity of a second operation later.

(b) When adequate examination is not possible without general anaesthesia and the eye is then found to be non-repairable:

(c) When coincident laceration of lids suitable for primary suture is present. If a disorganised eye is left, the resultant discharge will militate against primary healing of lid wounds, particularly those of lid margin.

(d) When there is much pain, as is sometimes the case with 
large uveal prolapses adhering to the lid margins, and often the case with large orbital foreign bodies.

(e) When panophthalmitis has already supervened,

The above constitute about 80 per cent. of all non-repairable eyes, and therefore except during excessive rush periods, very few are sent on unoperated.

The operation for non-repairable eyes has almost invariably been evisceration and excision of sclera, leaving a small frill. It would seldom be possible to do otherwise at this level, for the eyes that must be removed here are usually dirty, ragged ocular remnants, and enucleation would be difficult and have little advantage, quite apart from the danger, hypothetical or real, of meningitis. Occasionally the conjunctiva has been sutured over the stump after insertion of penicillin powder, but more often it has been left open. Reports from the base indicate that it might be possible to hasten socket healing by suturing conjunctiva in rather more cases provided they are carefully selected.

(II) Repairable.-Under this heading are included all cases which are anatomically repairable, whether they be-

(i) Eyes with no perception of even the brightest light;

(ii) Eyes in which mild sepsis is already present;

(iii) Eyes in which one knows that there is barely one chance in a hundred of their being of any value.

This is an extreme.policy and is only justifiable because one is working as part of a team and knows that the patient will not be allowed to run any risk of sympathetic ophthalmitis. The few cases which unexpectedly retain or attain some vision, even if only perception of light, especially if they happen to be bilateral, more than justify the very many cases which later lose the eye.

It is comparatively rare to find a man who has in his whole body only one wound of one globe. The majority of cases have perhaps one eyelid and eye, one arm and one leg wounded, or some similar combination. , Perhaps one eye severely and one mildly damaged. Perhaps multiple peppering of chest and face with one eye perforated and one with multiple corneal foreign bodies. It is therefore impossible to divide the cases into groups, but only the wounds. In the following description therefore it must be borne in mind that more than one of the wounds may be present in the same case.

Perforating wounds of the globe.-These are the commonest type of wounds requiring surgery, and may be divided into two groups :-

(a) Those produced by blast;

(b) Those produced by foreign bodies.

(a) Produced by blast.-This type of wound was not uncommon in air-raids particularly in cities where the blast 
occurred in confined spaces. With the lessening of aerial bombardment, at any rate in the field, they have become less common, but are still occasionally seen in men who have been very close to a shell, mortar, or grenade burst, but have not actually been hit by a fragment. Only eight such eyes have been seen of which three had already had the lens and a large amount of vitreous extruded, and were therefore worthless. The ruptures all occurred in the usual site i.e., nasally, and attempts were made to repair the others, but as the ruptures were mostly semi-circumferential little success was met with. Two of them retained perception of light up to a month after operation, but the end result has not been traced. As many as eight scleral sutures were used in one case. Three of the cases were bilateral.

(b) Produced by foreign bodies.-This group comprises the majority of cases operated on, and will therefore be dealt with in some detail. Slightly more than half the number (53 per cent.) had perforating wounds of the cornea or limbus, and slightly less than half (47 per cent.) had perforating wounds of sclera. In peace time corneal perforations predominate more markedly and the reason presumably is that in wartime the fragments producing these wounds are travelling at a higher speed, and therefore the lids offer less protection. It is certainly a fact that a higher percentage of cases is found in wartime with perforations of lid and globe. In this group only three cases occurred in which the injured man was wearing spectacles at the time of wounding ; i.e., less than 1 per cent. Since considerably more than 1 per cent. of front line troops wear spectacles it may be assumed that they afford some protection against a perforating wound of the globe even in wartime.

In dealing, with these cases the principle has been to attempt to remove all foreign bodies, all prolapsed tissue, and to close all open wounds at the first opportunity. There are of course many circumstances which make it advisable to deviate from this principle, and no hard and fast rules can be laid down. Exceptions to this principle have been made in the following type of cases :-

(a) In which a foreign body, possibly magnetic, is lying in a collapsed anterior chamber which is not reforming, and the lens is as yet undamaged. Any attempt to extract the foreign body will assuredly damage the lens. The better procedure is to close the wound with a conjunctival flap and wait till the anterior chamber reforms.'

(b) In which there is a foreign body in the lens, and a clean self-closing wound of entry. Whether magnetic or not, the foreign body is better left until the eye settles down, when it can be removed while the traumatic cataract is being dealt with.

(c) In which the wound is self-closing, there is no prolapse, 
and it is doubtful whether the foreign body is intra- or extra-ocular. Such cases can travel without harm and can well wait until they reach base for accurate localisation.

(d) The above are purely ophthalmic considerations. There must also be considered the general condition of the patient and his other wounds, particularly penetrating wounds of chest or head, severe burns, and cases which have already had one operation during the day. The moving of such cases to and from an operating theatre and possibly the giving of a general anaesthetic might cause one to sacrifice a life in order possibly to save an eye.

For those, cases in which operation is to be undertaken the preoperative measures have already been described.

\section{Operative measures}

Preparation of the field of operation consists in a good wash with soap and water followed by saline, cutting of the eye-lashes, and instillation of cocaine drops, whether or not a general anaesthetic is being given. In some cases, particularly mine injuries with the whole face spattered and burnt, it is advisable to shave the eyebrows, moustache if any, and as much of the scalp as is affected. No skin antiseptics are used.

\section{Scleral perforating wounds}

(a) Through and through.-It is rare for both entrance and exit wounds to be visible although a few such cases have been seen. More usually, the position of the exit wound is unknown, and its situation can only be guessed at from the position of the foreign body in X-rays.

No attempt is made to remove the foreign body, and unless both wounds are exposed or visible no attempt is made to repair the exit wound. The conjunctiva is dissected away from the entrance wound in such a way that it will be easy to pull it back as a flap over the scleral wound. (Diagram 1.) The edges of the scleral wound are cleaned and trimmed; usually with a knife which is easier than scissors, all prolapsed uveal tissue and vitreous

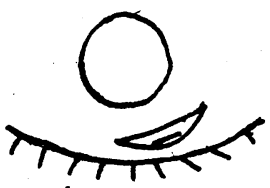

I.

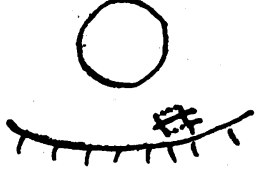

2 .

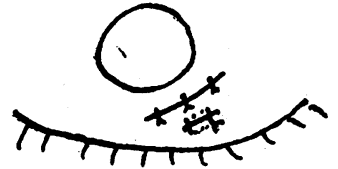

3.

abscissed, and the wound drawn together with scleral sutures. For this purpose, there is no doubt that Stallare's corneal needles on number 00 silk by " Mersutures," are by far the easiest to insert, 
particularly as the eye is already soft. For linear wounds an attempt is made to insert the sutures in the form of a square.or mattress stitch. (Diagram 2.) When the wound is circular with ragged edges this is not always feasible; and the wound must be closed however possible with the minimum wrinkling of the sclera. The conjunctiva is then drawn over the scleral wound in such a way that the two suture lines do not coincide. (Diagram 3.)

The foreign body which strikes the eye tangentially, lacerating the sclera is only a limiting example of a through and through wound, and presents no different problems.

(b) With retained foreign body.-In the majority of cases it is not possible to see the foreign body ophthalmoscopically on account of vitreous haemorrhage. However, with an open wound of the sclera the exact position of the foreign body is not of great importance since an attempt to remove it via the wound of entry will be made wherever it is. Less harm will result to the eye by removing it via the entry wound than by trying to localise it by the Skeoch ring 'method, close the entry wound, and open' the sclera in another place, all of which would entail extra manipulation in an already severely damaged and soft eye. It must here be stressed that this applies only so long as the entry wound is still open.

When attempting to remove the foreign body it is advisable to expose and clean the scleral wound, and insert the necessary sutures before abscissing the prolapse or bringing the magnet up to the wound. It sometimes happens that abscission of prolapse or extraction of a foreign body is followed by a flow of vitreous, and if the sutures are not already in place their insertion at this stage will certainly cause more vitreous to be lost.

My former practice was to try the Livingstone hand magnet in every case before trying the Haab magnet, but so many cases were found in which the former gave no response, and the latter extracted the foreign body without difficulty, that I have discarded the former. The Haab magnet is counterbalanced on a swivelling stand so that it can be manipulated into any position with ease and accuracy. In addition, a foot switch is far more convenient than a thumb button, as on the former. No attempt is made to cauterise the wound edges or the neighbouring choroid, partly because no diathermy apparàtus was available, but chiefly because the trauma of the perforation seems to produce an adequate scar, and although retinal detachments do occur it is not thought that they would be avoided by diathermising an area around the wound in a soft eye. Again, these remarks apply to the open wound case and not to the set operation of posterior route extraction of foreign body. 


\section{Corneal and limbal perforating wounds}

(a) Without retained foreign body.-These are usually caused by a fragment striking tangentially. This type of case is very similar to the peacetime injury by the "screw driver that slipped" or the knife blade accident. It presents no separate problems, and repair is by abscission of prolapsed tissue, and wound closure by a conjunctival flap.

(b) With retained foreign body. - When a single limbal perforation is present with a hole in the iris and little or no damage to the lens, it is frequently possible to draw the foreign body back along its track if it is magnetic. It can perhaps be removed by the entry wound or at least brought into the anterior chamber and removed by a keratome section. This method may result in a small anterior synechia, but this can be divided later.

When a single perforation is slightly nearer to the centre, there will probably be a hole in the iris or a cut in the pupil margin, a prolapse of iris and lens capsule, a completely opaque lens, and possibly the anterior chamber will be absent. In these cases extraction via entry wound or anterior route is not advisable and primary operative measures should be confined to abscission of any prolapse, and closure of the entry wound to ensure reformation of the anterior chamber. The foreign body should be left for posterior route extraction later, after X-ray localisation.

If a single perforation occurs in the pupillary, area, damaging the lens, the method of approach will depend on :-

(1) The condition of the anterior chamber;

(2) The state of swelling of the lens, and

(3) The situation of the foreign body on X-rays.

Absence of the anterior chamber is a definite contraindication to anterior route attempt. If the anterior chamber is full of soft lens matter and the foreign body is somewhere amongst it, as shewn by X-ray, it is justifiable to remove it anteriorly. If the foreign body is shewn to be in the posterior two thirds of the globe it should be removed by the posterior route.

In those cases where there is no swelling of the lens, the anterior chamber is present, the pupil can be well dilated; and the foreign body can be seen or localised somewhere behind the lens, the method of extraction depends on the personal choice of the surgeon. Details of the classical anterior and, posterior route extractions are too well known to be included here, but there is one point worth mentioning. In making the keratome section for the anterior route extraction of foreign body, it should be commenced two or three millimetres inside the limbus. The inner lip of the incision will then be about four millimetres from the angle of the anterior chamber. This will make it easier to 
avoid prolapse of iris and/or loss of aqueous. Should the anterior chamber be lost during the making of the incision no attempt should be made to remove the foreign body until it has reformed or been refilled.

Lastly, there are the cases with multiple corneal perforations. Usually there is also prolapse of iris, lens matter and vitreous, and perhaps gravel, sand, or clay soil driven right through the cornea into the anterior chamber. They are almost always mine injuries, frequently bilateral, and the results are disappointing: In spite of such gross damage to the anterior section of the eye they frequently have perception of light with accurate projectlon. I have tried doing the minimum to the eye and also doing the maximum. The minimum consists in washing the eye out, putting in atropine, penicillin, and a pad and bandage and leaving it to recover or not, as best it can. The maximum consists in abscissing all prolapse, removing foreign bodies or debris from the anterior chamber with a scoop, and from the cornea with a needle, refilling the anterior chamber with penicillin and covering the whole eye with a conjunctival flap. My impression is that there is very little to choose between the results of the two methods, so that a middle course probably gives the eye the best chance. A number of such cases have settled down without infection, even with visible particles of stone in the anterior chamber.

There are two subjects about which a word must be said, namely, conjunctival flaps and penicillin.

\section{Conjunctival flaps}

There are very few cases in which a complete purse-string conjunctival cover is necessary, and it has the great disadvantage that the condition of the eye underneath it cannot be seen. This disadvantage is bad enough when the case is under the care of one surgeon, but when a case is being passed from one surgeon to another it becomes even worse. It is therefore better to avoid it whenever a visor flap will serve the same purpose, as it usually will. In cutting the flap, care should be taken to make it as thin as possible. Not only is a thin flap more elastic, but it does not tend to adhere to the wound so much as a full thickness flap. It frequently happens - particularly in mine injuries - that the conjunctiva is damaged in several places. When the flap is dráwn over the corneal defect it can usually be arranged that an intact piece of conjunctiva covers the actual defect, and there is no need to mend the holes.

I have found that when dissecting a flap the use of a sharp scalpel or Bard Parker blade, instead of scissors, not only facilitates the procedure ; but enables one to obtain slightly more conjunctiva near the corneal margin. 


\section{Penicillin}

The use of penicillin in ocular injuries is still in the experimental stage and it is not possible as yet to give any statistical results proving its value. There are, however, some indications which are worth mentioning.

(1) Since January, 1944, penicillin sodium drops or cream have been used as routine at the end of every operation, and at every post-operative dressing. Prior to January, 1944 , penicillin was not used. In the pre-penicillin era six cases out of 132 (4.5 per cent.) developed panophthalmitis in the first few days after operation. Since January, 1944,3 cases out of 214 (1.4 per cent.) have developed panophthalmitis in the first few days.

(2) It has been found that the stickiness and discharge usually present at post-operative dressings has been minimal or absent, since the use of penicillin.

(3) Sodium penicillin solution ( 1,000 units per c.c.) has been introduced into the anterior chamber during operation in 20 cases, most of which have been mine wounds with dirt and debris in the anterior chamber. It is not considered that this has had any markedly favourable effect on the progress of the case. In 6 cases sodium penicillin, minims 5 (1,000 units per c.c.) has been injected into the vitreous along the suspected course of stone intraocular foreign bodies. In three of these cases, panophthalmitis supervened as expected. Of the other three, one gradually developed endophthalmitis, but the other two retained an eye with accurate projection of light up to a fortnight, and a month respectively. Since they were both civilians further follow up was impossible.

My impression so far therefore is that penicillin is of considerable value as routine in the conjunctival sac, of some value as a vitreous injection, but of no value as an anterior chamber wash out or injection. It has been proved experimentally that it diffuses readily throughout the vitreous by direct injection, but not at all by intramuscular injection. Sub-conjunctival injection is being tried, throughout this theatre.

\section{Non-perforating wounds of the globe}

(1) Corneal foreign bodies. - These vary considerably in size, number, and depth. It is often impossible to remove more than a few at one session. Some will work their own way out. Some will remain there always without causing any trouble. The ones which should receive attention first are those projecting above the level of the cornea, and those in the pupillary area of the cornea, where residual scarring will cause most disability.

(2) Scleral foreign bodies.-Although these per se are not 
urgent or dangerous it is frequently difficult to tell whether they are perforating or not. If there is any doubt the track should be explored and the sclera examined.

\section{Wounds of soft tissue}

Lid Wounds.-Provided that there is no tissue loss, all but the very dirtiest of lid wounds can be sutured with the aid of penicillin powder. It is surprising how often a lid wound which appears dirty will heal by first intention when thoroughly washed and sprayed with penicillin powder. It is a distinct advantage to suture lid margin wounds straight away whenever possible, for they heal with less of a notch than if left till later.

If there is any loss of lid tissue, either skin or deeper tissue it is better to clean them thoroughly, excise nothing at all, spray with penicillin, and send on to a Maxillo-facial unit. It is sometimes advisable in extensive wounds to put in two or three temporary sutures, leaving long ends, merely to hold the parts in approximately the right position.

\section{Compound fractures around the orbit}

It is quite common for the missile which destroys an eye to produce a fracture of the orbital walls, perhaps involving one of the air sinuses.

The foreign body is often firmly embedded in bone, and unless it is easily accessible it is better to leave it alone and confine one's activity to toilet of the wound, removing only such soft tissue as is non-viable and only such bone chips as have no periosteal attachment. One of the commonest cases is that of a foreign body passing through the lid, eye and floor of orbit, and coming to rest in the antrum. Having removed the eye it is worth while putting the magnet up to the defect in the orbital floor, when, in quite a number of cases the foreign body is easily removed. Very few cases develop an empyema of the antrum, and it is possible that sulphanilamide is responsible for this.

Only two cases have been seen in which an orbital foreign body was considered to require immediate removal. In both cases the eye was present and was not perforated, and recovery of function was expected. The foreign bodies were large and were causing displacement of the eye, limitation of movement, and pain. The first one was a shell fragment, about the size of a lump of sugar, and had entered through the cheek, on the opposite side. It was lying in the upper outer part of the orbit and was removed via conjunctiva and Tenon's capsule. The second was a Tommy gun bullet which had entered the external canthus, and become embedded in the centre of the floor of the orbit with the round 
nose uppermost. This was removed by the wound of entry after canthotomy and medial retraction of the orbital contents.

Fractures of the upper part of the orbit, so severe as to expose or open the dura are better dealt with by the Neurosurgical unit.

\section{General considerations}

One of the most surprising facts is the very low incidence of sepsis either as panophthalmitis, hypopyon ulcer, or cellulitis. In spite of the dirty conditions of the wounds, and the far from ideal conditions of asepsis obtaining in a field operating theatre, sepsis was extremely rare.

In my opinion the reasons for this are :-

(1) The healthy state of the front line troops;

(2) The routine administration of sulphanilamide at the earliest opportunity to all wounded men ;

(3) The routine use of penicillin in all eye injuries;

(4) The minimum number of changes of dressings.

One of the chief differences between the wartime intra-ocular foreign body and the peace time one is the almost constant presence of vitreous haemorrhage in the former. This may be caused by the higher velocity of the fragment, or the blast that always accompanies it. It adds to the severity of the wound and consequently lessens the chance of recovery of good vision.

The statistics given below will give a good idea of the type of cases and their relative frequency. No attempt is made to assess results.

440 consecutive cases of battle casualty eyes requiring major operations October, 1943, to October, 1944.

(1) Missile accounting for wound.

\begin{tabular}{|c|c|c|c|c|c|c|}
\hline Shell & & & & 102 & $=3$ & \\
\hline Mine & $\ldots$ & $\ldots$ & $\ldots$ & 68 & $=$ & 20 \\
\hline Mortar & $\ldots$ & $\ldots$ & & 57. & $=1$ & $16 \cdot 7$ \\
\hline Grenade & $\ldots \quad \ldots$ & $\ldots$ & $\ldots$ & 38 & $=1$ & $11 \cdot 1$ \\
\hline Unknown & Missile & $\ldots$ & & 30 & $=$ & $8 \cdot 8$ \\
\hline Bullet & $\ldots \quad \ldots$ & $\ldots$ & $\ldots$ & 16 & $=$ & 4.7 \\
\hline Booby Tra & ap & $\ldots$ & $\ldots$ & 15 & $=$ & $4 \cdot 4$ \\
\hline Aerial bon & $\mathrm{mb} \quad \ldots$ & $\ldots$ & $\ldots$ & 7 & $=$ & 2 \\
\hline Hammer 8 & \& chisel & $\ldots$ & $\ldots$ & 6 & $=$ & $1 \cdot 8$ \\
\hline
\end{tabular}

(2) Eye irreparably damaged and removed $59=28.7$ per cent.

(3) Number of cases in which brain was involved $20=6$ per cent.

(4) Number of cases in which accessory sinuses were involved $40=12$ per cent.

(5) Number of eyes in which panophthalmitis supervened a few days after operation $9=2.5$ per cent. 
(6) Of 208 repairable perforating wounds : scleral $97=47$ per cent.; limbal and corneal $109=53$ per cent.

(7) Of these 208 eyes the number with probably no intra-ocular foreign body (including through and through wounds) 41 $=20$ per cent.

(8) Of these 208 eyes the number probably with intra-ocular foreign body $167=80$ per cent.

(9) Number of eyes in which intra-ocular foreign body was removed by magnet $72=43$ per cent.

(a) Anterior route $12=16.7$ per cent.; (b) Posterior route $10=14 \cdot 4$ per cent.; $(c)$ Wound of entry $50=69$ per cent.

(10) Number of eyes in which intra-ocular foreign body was removed by means other than magnet $18=10.8$ per cent.

(11) Number of eyes in which probable intra-ocular foreign body was not removed $77=46$ per cent.

(12) Number of cases in which intra-ocular foreign body was present, but in which no attempt at removal was advisable $6=3 \cdot 6$ per cent.

\section{Summary}

(1) A description is given of the working of a Mobile Ophthalmic $\mathrm{Lnit}$ in the Mediterranean Theatre of War.

(2) Surgical procedures adopted in dealing with various eye injuries are outlined.

(3) An analysis of the major operations performed during a year in the forward areas is detailed, showing the relative frequency of various types of eye injury.

In conclusion, I would like to thank, first, my sergeant and four men, without whose tireless efforts, cheerfulness, and close co-operation, both while working and while moving, little could have been accomplished; secondly, the Adviser in Ophthalmology A.F.H.Q., for his continuous support in keeping this unit at all times fully equipped with everything that could be required, and for his comments and helpful suggestions on cases sent back; thirdly, a number of C.C.S. Commanders who spared no pains to accommodate what must have been to them a rather troublesome attached unit; and finally the D.M.S., A.F.H.Q., for his interest in the unit, and for permission to publish this paper.

Abbreviations.

C.C.S. = Casualty clearing station.

A.D.S. = Advanced dressing station.

M.D.S. = Main dressing station.

F.D.S. = Field dressing station.

R.A.P. $=$ Regimental Aid Post.

R.M.O. = Regimental Medical Officer. 